\title{
Integración de la estructura organizacional en coordinaciones de postgrados en universidades del estado Zulia - Venezuela
}

\author{
Reynier I. Ramírez ${ }^{1 \star}$, Joiner D. Torres ${ }^{1}$, María V. Velilla ${ }^{1}$ y Nelson D. Lay ${ }^{2}$ \\ (1) Universidad de la Costa, Dpto. de Ciencias Empresariales, Atlántico - Colombia \\ (correo-e: rramirez13@cuc.edu.co; jtorres75@cuc.edu.co; mvelilla@cuc.edu.co). \\ (2) Universidad Andres Bello, Escuela de Psicología, Viña del Mar - Chile (correo-e: nelson.lay@unab.cl). \\ * Autor a quien debe ser dirigida la correspondencia.
}

Recibido Jun. 18, 2021; Aceptado Ago. 18, 2021; Versión final Sep. 20, 2021, Publicado Dic. 2021

\begin{abstract}
Resumen
El estudio describe la integración de la estructura organizacional en las coordinaciones de postgrados de universidades del estado Zulia. Se usó un enfoque cuantitativo, de tipo descriptivo, no experimental, y diseño transeccional, utilizando como unidades de observación 104 coordinadores. Se aplicó un cuestionario validado por diez expertos, con una confiabilidad de $\mathrm{rtt}=0,857$ calculada con el método Alfa Cronbach. Los resultados muestran la presencia de los indicadores que integran la estructura organizacional, siendo el de mayor puntaje el ideológico, seguidamente de lo orgánico-estructural, el normativo, tecnológico, desfavoreciendo lo psico-social. Se concluye que la estructura organizacional en las coordinaciones de postgrados, integran la cultura institucional y dimensiones de su doctrina. Además, estandarizan el comportamiento organizacional como respuesta operante en la consolidación de metas.
\end{abstract}

\section{Integration of the organizational structure in graduate coordination at universities in the state of Zulia - Venezuela}

\begin{abstract}
The study describes the integration of the organizational structure in the graduate coordination of universities in the state of Zulia. A quantitative approach, descriptive, non-experimental, and transactional design was employed, using 104 coordinators as observation units. A questionnaire was applied and validated by ten experts, with a reliability of $r t t=0.857$ calculated with the Cronbach Alpha method. The results show the presence of the indicators that make up the organizational structure, the ideological being the one with the highest score, followed by the organic-structural, the normative, the technological, disfavoring the psychosocial. It is concluded that the organizational structure in graduate coordination integrates the institutional culture and dimensions of its doctrine. It also, standardizes organizational behavior as an operative response in the consolidation of goals.
\end{abstract}

Keywords: organizational structure; graduate studies; strategic management; technological resources 


\section{INTRODUCCIÓN}

Debido a la alta demanda social inmersas en el fenómeno de la globalización, hoy en día los institutos de educación superior buscan crear profesionales innovadores, competitivos y estratégicos, teniendo en cuenta la calidad e internacionalización donde predomina la autoevaluación y acreditación como factor imprescindible en las políticas de gestión universitaria. Las universidades se encuentran en un cambio estructural de la gestión ejecutiva y perfiles administrativos, llevando a los líderes y personal a realizar actividades que hacen parte de la estructura universitaria, para promover conocimiento y nuevas ideas administrativas - académicas. Es así, como los constantes cambios del entorno, sistemas de información, economía y estrategias gerenciales, han llevado a las universidades a originar novedosas estrategias adaptativas y aprendizaje que impulsen a la sustentabilidad, adaptándose así a los requerimientos del entorno local, regional, nacional e internacional. Según Ramírez et al., (2019), Villalobos et al., (2019) y Sukier et al., (2020), las estructuras organizativas se incrustan en los patrones de progreso para ajustarse a las metodologías administrativas que les permiten soportarse en la innovación, reducir la burocracia y lo piramidal, disminuyendo tiempos de espera para la toma de decisión, teniendo como objetivo mejorar la competitividad y permanencia en el mercado.

Entre las acciones necesarias para adaptarse a la estructura de las instituciones de educación superior, se puede nombrar la implementación de propuestas pertinentes en los programas de postgrado, donde se evidencie el desarrollo de áreas de conocimientos emergentes, llevando a cabo un aprendizaje idóneo en los diferentes niveles de postgrados (especialización, maestría, doctorado y post - doctorado), fortaleciendo las competencias técnicas y genéricas del talento humano, logrando obtener una mano de obra calificada para cumplir con la demanda real del sector productivo. Con respecto a, los proyectos de postgrado añaden coherencia a la interacción de la educación, generan información e innovaciones en los procesos y sistemas actuales, respondiendo a los problemas sociales que se presentan dentro de las comunidades. Para la idealización y desarrollo de los proyectos de postgrado, se han establecido procedimientos y modalidades institucionales que intentan reaccionar a las necesidades que presentan los graduados incorporados en el mercado laboral.

Al respecto, la estructura organizacional establece el sistema jerárquico de la universidad a fin de administrar sus recursos, elementos y capacidades, impactando responsablemente sus áreas funcionales tanto administrativas como operativas, con miras a la sustentabilidad para la consolidación de metas, adaptándose a las transformaciones del mercado y generación de valor. Según Holbeche (2018) y Sergeeva et al., (2021), manifiestan que el nivel de exigencia de los administradores académicos varía y se adapta según los cambios, debido al desarrollo de asociaciones educativas de espacios que favorezcan al desarrollo del pensamiento y visión completa desde lo individual, hasta lo grupal, enfocados al cambio y avances de los objetivos, necesidades y cualidades de los miembros, desarrollando soluciones que den respuesta a los problemas presentes en las sociedades, estableciendo estándares de valor y competencia en la administración estratégica.

En cuanto a la estructura organizacional académica en Latinoamérica Braßler (2020), establece dos modelos básicos, el primero el de las facultades, orientado a los resultados, establece que cada facultad tiene la responsabilidad del diseño y actualización del plan de estudios y malla curricular profesional; y el segundo por departamentos, encaminado a los procesos, establece que los departamentos reúnen a los docentes e investigadores de la universidad especializados en una misma disciplina, creando un conjunto de asignaturas pertinentes a su ámbito académico disciplinar y son cursadas por estudiantes de distintas carreras. Las universidades actuales están optando por la implementación de una estructura organizativa departamental con el objetivo de crear una vinculación de programas, así como el aprovechamiento de los recursos de la universidad promoviendo la investigación y el desarrollo en los campos de estudio universitarios al reunir docentes e investigadores de una misma disciplina.

Annía et al., (2019), manifiestan que en Venezuela la educación superior se encuentra en una etapa crucial de búsqueda donde los nuevos modelos de gerencia son ideales para controlar constantes cambios tecnológicos como el de la comunicación, pretendiendo la interrelación en cualquier lugar del planeta. Desde este punto de vista, la educación superior en las instituciones de Venezuela persigue la reestructuración para lograr su continuidad en el tiempo. Para Brunet et al., (2021), la educación superior en Venezuela puede: (1) verse motivada por diferentes puntos de vista sobre la formación académica, la cual ha permitido a la sociedad aproximarse a la globalización con calidad, desarrollo, visión y ordenamiento de estándares, (2) permitir la adaptación al cambio, enfocarse en el juicio, evaluación, colaboración y cooperación de los miembros de cada área que integran las universidades y agentes externos que logran tener una relación con productos o servicios ofertados por la educación superior, liderando los procesos de formación y administrar los recursos que permitan una adaptación y transformación; y (3) contar con la capacidad para asumir el compromiso y conductas, simplificando estructuras jerárquicas lineales antiguas; por su parte Santangelo et al., (2021) y Salmela et al., (2021), exponen que las actividades que se dan de manera grupal entre los individuos, son 
algo útil para poder crear nuevas ideas y hacer propuestas que den origen a un estructura nueva desde y hacia las organizaciones, implementando herramientas y métodos que faciliten la adaptación y comunicación en la estructura organizacional.

En las organizaciones de educación universitaria venezolanas, particularmente los programas de postgrado objeto de este análisis, cuentan con cambios académicos y administrativos, áreas curriculares de todos los programas académicos de postgrado y capacidad administrativa de los niveles estructurales; originando requerimientos, actividades intra e interpersonales de relación en la disposición vivencial, social y laboral de estas instituciones. En tal sentido, es propio observar que quienes gerencian estos programas de postgrado, en el desarrollo de su rol, así como en el modo operacional, están inmersos en nudos críticos operativos, que desmejoran la gestión organizacional de las casas de estudio, experimentando cambios estructurales, impactando las mallas curriculares de todos los programas académicos de postgrado y capacidades administrativas estructurales; dando origen a nuevos requerimientos, actividades de desarrollo personal e interpersonal para lograr obtener mayores niveles de bienestar laboral y social en las instituciones de educación superior. Los encargados de gestionar dichos programas de postgrado, en el desarrollo de su función están inmersos en múltiples y complicados nudos operativos, que ralentizan la gestión organizacional de las ramas de estudio.

Partiendo de estas ideas, Menon y Suresh (2021), señalan que algunos gerentes académicos a cargo de los programas de postgrado asumen el cargo de coordinador sin un compromiso claro para establecer con el talento humano a su cargo perspectivas de trabajo sistémicas. Este argumento induce a la implementación o inclusión de la planeación, organización, dirección y control dentro de las estructuras organizativas de las coordinaciones académicas para establecer el lineamiento estratégico de los objetivos propuestos a la consecución de metas, así como su seguimiento y evaluación para una mejora continua y adaptable a los cambios. Villalobos y Ramírez (2018), han revisado en sus estudios, la falta de estimación que le brindan los expertos que ejercen la docencia, quienes están ejecutando su función desde el ámbito gerencial, a los puntos referidos de las ventajas académicas y expertos que brindan al personal el proceso de colaboración protagónica, trabajo en grupo, la pertinencia organizacional, como consecuencia de la instauración de un sistema democrático, con base en el compromiso y responsabilidad.

De la misma forma Inciarte (2012), afirma que el desconocimiento u olvido de los gerentes educativos, desfavorece la mejora de los procesos técnicos de la gestión pedagógica, procesos de aprendizajes e interacciones en grupo encaminadas a la creación de proyectos creativos e innovadores que conecten a la organización con la estructura social. Tomando como referencia los factores antes mencionados, se pueden citar puntos críticos en el detrimento de la estructura organizacional en las coordinaciones de postgrados de universidades del estado Zulia, como el enfoque individualista de los líderes pretendiendo alcances colectivos o sociales, con fines particulares, limitados y desconectados del interés colectivo, falta de atención a las inquietudes individuales de la exigencia estudiantil respecto a los programas de postgrado. Esta comunidad estudiantil busca mejorar sus competencias para satisfacer sus necesidades personales y laborales, desmejoras de los planes curriculares con propuestas transversales, las cuales tienen que ser cumplidas como requisitos por todos los cursantes, impidiendo la inter y transdisciplinariedad del desarrollo académico y científico demandado por la nueva era del conocimiento, en donde lo innovador, tecnológico y emprendedor es prioridad en el mundo.

Esta problemática es producto de una gestión autocrática, organización centralizada y un modelo académico desarticulado de la razón de ser universitaria y necesidades del entorno, generando el deterioro de la consolidación de los principios corporativos universitarios, que afectan negativamente la estructura organizacional en las coordinaciones de postgrados de las universidades del estado Zulia, evidenciando un impacto social, ambiental y económico incierto en las funciones sustantivas de la gestión universitaria, implicando un interés individualista en la administración, una falta de planificación, desaprovechar los recursos y estructura organizativa. Además, İpek y Karaman (2021), señala como problema fundamental los presupuestos y el funcionamiento universitario, puesto que no existe una correspondencia con el producto o servicio que recibe la sociedad, es decir se refleja ineficiencia en la gestión universitaria, bajo los sistemas cambiantes de las estructuras organizacionales, los cuales deben ser ágiles, con la capacidad de adaptarse a los avances y exigencias de mayor productividad y eficiencia de los recursos disponibles. La falta de comprensión de estos factores administrativos y académicos dificultan a los estudiantes ser competitivos en el mercado laboral, disminuyendo el impacto social de los profesionales de postgrado y comprometiendo la sostenibilidad de la institución en un largo plazo.

Es necesario resaltar que los gestores educativos permanecen ocupados la mayor parte del tiempo en funciones operativas rutinarias como realizar horarios, informes o hacer inscripciones, dejando a un lado la gestión de lineamientos estratégicas involucradas y basadas en las fortalezas de cada programa académico, por todos los factores mencionados anteriormente se dificulta la creación científica, tecnológica y social. Esto 
provoca como consecuencia el desconocimiento y efectividad del rol de gerente educativo, así como sus capacidades administrativas destinadas al buen manejo de las instituciones de educación superior, motivo por el cual se plantea la siguiente interrogante ¿Cómo está integrada la estructura organizacional en las coordinaciones de postgrados de universidades del estado Zulia?

\section{OTROS ANTECEDENTES}

La educación superior se enfrenta a retos globales, priorizando entonces en la articulación de la estructura organizacional. De esta manera, se puede decir que la educación profesional está en una transformación de sus estructuras, competencias y de gestión, enfocándose en los ejercicios reflexivos de la ecología universitaria (económica-ambiental-social), con la finalidad de incluir aportes y construcciones del concepto de universidad sustentable, donde el educando pueda autogestionar su vida de manera responsable, pertinente y resolutiva en el entorno donde se desarrolle. Los cambios repentinos de la globalización han impulsado la tecnología, sistemas de información, economía y políticas gerenciales, esto ha procurado en la estructura organizacional de educación superior desenvolver procesos de transformación competitivas, respondiendo así con servicios de calidad para generar satisfacción al profesional, desembocando en las universidades la implementación de estrategias de supervivencia, para mantenerse y responder a las necesidades del mercado.

Según el concepto de estructura organizacional, Ramírez et al., (2020) y Sukier et al., (2020a), lo definen como un modelo de relaciones entre los elementos o parte de la organización, influyendo en las operaciones reales y comportamiento de la institución. Es un sistema sociotécnico abierto integrando objetivos y valores, técnico, psicosocial, estructural y administrativo, originando un propósito que debe alcanzar una organización. Ríos et al., (2020) y Saarikko et al., (2020), manifiestan que buscan establecer relaciones entre sus componentes internos denominados subsistemas, siendo un sistema o todo unitario compuesto por dos o más elementos interdependientes con ciertos límites vinculados a un suprasistema. Para Bello-Pintado y Bianchi (2018) y Ryttberg y Geschwind (2021), las estructuras avanzadas de la empresa moderan positivamente la relación. Al mismo tiempo Belloso (2013), plantea que el tipo de estructura organizativa en las universidades venezolanas son burocráticas, departamental y sencilla. Las estructuras organizacionales en las universidades de Venezuela guardan un estilo tradicional, suelen irse más hacia instituciones cerradas, sin embargo, se notan en esta década, inicios en la búsqueda de las relaciones con el ambiente.

Es así como la estructura organizacional se incluye en las tendencias al cambio para adaptarse a los cambios y nuevos enfoques gerenciales que podrán apoyarse en la tecnología, disminuyendo al máximo lo piramidal, tiempo al momento de tomar de decisiones en función a la eficiencia y productividad. Las instituciones de educación superior están generando esfuerzos para la transformación y dar respuesta a la contemporaneidad, pero aun así se presentan dificultades para su conclusión a corto plazo (Gomera et al., 2020). Al respecto, se puede decir que la educación superior en Venezuela busca trascender nuevos paradigmas para liderar acelerados cambios tecnológicos, científicos y sobre todo la comunicación informatizada, llamando a replantear nuevas dimensiones que hagan vigentes el cumplimiento pertinente social y científico, enfocándose en (1) nuevas visiones de la educación, como una manera que permita a la sociedad acceder a la transferencia y gestión del conocimiento con calidad, adaptándose a los retos globales; (2) crear una capacidad hacia el cambio, basándose en la consulta, opinión comprometida, cooperación y colaboración de cada uno de los miembros de la comunidad educativa para el fortalecimiento de su capacidad de integración y transformación; (3) el compromiso para generar nuevas conductas de aceptación sobre la eliminación de antiguas estructuras jerárquicas, dando paso a originales tendencias emergentes de gestión estratégica de talento humano; e (4) incrementar estrategias que posibiliten la apropiación de estas estructuras con un enfoque sustentable. En este orden de ideas la estructura organizacional, es un sistema que establece los niveles de actividades académica - administrativa, dada por un modelo de gestión que cada institución usa para la realización de sus funciones sustantivas, mediante los indicadores ideológico, orgánico - estructural, tecnológico, psico social, y normativo, desarrollados a continuación.

El indicador ideológico, promueve un acto simbólico que determina una conducta tanto individual como grupal, establecidos en la filosofía institucional, valores, creencia e ideas. Dixon-Fowler (2020), muestra como al influir la ideología por medio de unos factores psicológicos a los cuales denominó TIP (contratos psicológicos infundidos), se pueden aumentar las conductas ciudadanas, fortalecer la identidad, aumentar la satisfacción laboral y mantener una percepción y reputación públicas positivas. Respecto a lo orgánico-estructural, hace referencia a la forma en la que se organizan y estructuran las actividades, relacionado con la autoridad y niveles de comunicación, determinando su constitución, puesto jerárquico y procedimientos. Según Modenov (2018), para garantizar la seguridad económica la estructura organizativa debe abandonar su enfoque funcional y utilizar un enfoque de procesos, disminuyendo el número de unidades estructurales, mejorando la eficiencia de la gestión. 
El indicador psicosocial, se orienta por la salud física, mental, psicosocial y social del talento humano, promoviendo el comportamiento interpersonal ya sea grupal o individualmente, considerando la motivación, liderazgo, sistema de esfuerzos y situación económica tanto del talento humano como de las organizaciones. Según Mihaljević (2020), en el proceso de modernización de la educación de doctorado basándose en un modelo causal de desempeño y cambio organizacional demostró que el entorno externo, la cultura organizacional y estructura, tuvieron una profunda influencia en la consolidación de la gestión del cambio durante la modernización de la estructura organizacional. Por consiguiente, el grado de importancia de este factor dentro de la integración de la estructura organizacional en las coordinaciones de postgrado universitarias. El indicador tecnológico, establece el conjunto de recursos, procesos y herramientas de información y comunicación que se incorporan a la estructura organizativa, según Marín (2020) el núcleo central para el aseguramiento de la calidad en la educación superior está dado por los sistemas de autoevaluación y el uso de la tecnología de la información. Lo normativo, establece el conjunto de reglas que tiene relación entre sí, algunos factores de análisis característicos denotan los contratos colectivos, leyes orgánicas y preceptos dictatoriales interiores de trabajo. Para Ríos et al., (2019), este indicador está asociado con la reglamentación que pretende regular la actividad misma de la institución. Algunos factores que caracterizan este indicador son los contratos colectivos, leyes orgánicas y reglamentos interiores de trabajo. En la tabla 1, se establecen los aportes para los indicadores antes mencionados de acuerdo con las teorías revisadas que exponen y describen la integración de la estructura organizacional, sistematizando la operacionalización de la variable y estableciendo criterios de análisis.

Tabla 1: Aportes de los indicadores que describen la integración de la estructura organizacional

\begin{tabular}{|l|l|}
\hline Indicadores & Aportes \\
\hline Ideológico. & $\begin{array}{l}\text { Fortalece la cultura corporativa y establece un grado democrático, materializados en el } \\
\text { comportamiento laboral grupal y percepción del personal. }\end{array}$ \\
\hline $\begin{array}{l}\text { Orgánico- } \\
\text { Estructural. }\end{array}$ & $\begin{array}{l}\text { Articulación entre las metas y composición, propiciando la flexibilidad, reduciendo la } \\
\text { burocratización y nivel de descentralización de los procesos de administración, así como la } \\
\text { posibilidad y suficiencia de la comunicación. }\end{array}$ \\
\hline Tecnológico. & $\begin{array}{l}\text { Incorpora la innovación y recursos digitales en los procesos y métodos de labor institucional, } \\
\text { grado de especialización de estos, interdependencia, interdisciplinariedad, efectividad y orden } \\
\text { organizacional. }\end{array}$ \\
\hline Psico-social. & $\begin{array}{l}\text { Promociona el desarrollo del personal, identificando los intereses y objetivos institucionales, para } \\
\text { la calidad de vida sustentable del talento humano. }\end{array}$ \\
\hline Normativo. & $\begin{array}{l}\text { Establece la regulación y adecuación, de los aspectos reglamentarios para su cumplimiento, } \\
\text { procurando el deber ser de los procesos y adecuado comportamiento en la institución. }\end{array}$ \\
\hline
\end{tabular}

Al identificar estos indicadores se logra analizar de forma práctica los elementos característicos de la estructura organizacional, su correcta articulación mejora la integración de esta. Resulta una ventaja competitiva para las universidades que se enfrentan al entorno globalizado y cambiante actuales, utilizar estos indicadores sistematizados, los cuales representan un factor clave para el proceso de consolidación de la estructura, crear una ideología en la cual todos los individuos pertenecientes al sistema educativo de la universidad se basen para orientar sus conductas y decisiones, estableciendo una cultura institucional sólida alineada con los objetivos estratégicos y filosofía organizacional que generan valor a la institución. Al entender la ideología y orientación de la visión institucional se comprende el horizonte académico que buscar, ayudando al talento humano a desarrollarse, relacionarse con la estrategia de las universidades, armonizar su entorno, administrar estratégicamente los recursos y capacidades, potencializar las dimensiones psicosociales, adaptándose a los cambios y optimizando sus capacidades de respuestas.

Actualmente se requiere que las estructuras organizacionales sean ágiles, capaces de adaptarse a las necesidades del mercado, abandonando ideas obsoletas de modelos burocráticos con demasiado peso jerárquico, invitando a los líderes a salir de lo convencional e implementar estructuras flexibles y ágiles las cuales representan mayor productividad, tienen una mayor capacidad de respuesta al cambio, y manejan menores tiempos de espera para la toma de decisiones, logrando de esta forma mayores niveles de eficiencia. En cuanto a lo digital es importante estudiar el impacto creciente de las tecnológicas de la información y comunicación (Tics) en las organizaciones, estas representan herramientas y recursos necesarios que garantizan la conectividad e información, aprovechada para establecer enlaces externos e internos en las instituciones, procurando accesibilidad, internacionalización, sistematización y procesamiento simultáneo de procesos y redes administrativas-operativas, logrando aumentar la eficiencia, productividad, alcance y calidad de la institución, otra ventaja que representa este factor es la innovación que estimula la creatividad, optimización de los procesos pedagógicos de enseñanza - aprendizaje, investigación, extensión y gestión universitaria. Respecto a lo normativo, establecen en las organizaciones reglas necesarias para mantener el orden, regular los procesos y ejercer un mayor nivel de control sobre las operaciones y relaciones personales institucionales, regulando conductas laborales. 


\section{METODOLOGÍA}

Se orienta por el paradigma cuantitativo, de tipo descriptivo, no experimental con un diseño transeccional. El proceso para consolidar el objetivo de la investigación se estableció bajo (1) el diagnóstico de la realidad problemática respecto a la variable de estudio en la población seleccionada, según los indicadores ideológico, orgánico-estructural, tecnológico, psico-social, y normativo, permitiendo desarrollar el planteamiento del problema y formulación de la hipótesis, (2) elaboración de los referentes teóricos que fortalecen tanto la variable de estudio (gestión organizacional), como sus dimensiones e indicadores (organización: funciones, estructura organizativa, actividades a ejecutar; planificación: actividades planeadas, conocidos de objetivos organizacionales, plan de acción para alcanzar objetivos; recursos: presupuesto para el funcionamiento, disposición de espacio físico, disposición de recursos materiales de oficina; y resultados: valoración de acciones finales, mecanismos de reporte del desempeño, efectividad en el resultado), (3) establecimiento del marco metodológico, especificando los procedimientos científicos para materializar la investigación, y la (4) presentación y análisis de los resultados, dando a conocer los hallazgos de la interpretación de los datos, y así poder generar las conclusiones; todo esto bajo la orientación teórica de Tamayo y Tamayo (2012), Arias (2012) y Hernández, Fernández, y Baptista, (2014).

En este estudio participaron ciento cuatro (104) coordinadores del departamento de postgrado de La Universidad del Zulia (LUZ), Universidad Privada Dr. Rafael Belloso Chacín (URBE), Universidad José Gregorio Hernández (UJGH) y la Universidad Pedagógica Experimental Libertador (UPEL), ubicadas en la República Bolivariana de Venezuela, en el estado Zulia, los criterios para la elección del grupo poblacional corresponde a su razón social por ser instituciones conducidas por el ministerio del poder popular para la educación universitaria, ciencia y tecnología, las cuales disponen de unidades gerenciales en su estructura jerárquica, departamentos o dependencias, cantidad de coordinaciones y servicios mediante el vicerrectorado de investigación y postgrado; necesario para la obtención del objetivo propuesto en la investigación, permitiendo realizar un censo poblacional, tomando en cuenta todos los sujetos de la población, caracterizados por ser profesionales, mayores de edad, con un contrato indefinido y todos bajo el cargo homólogos; indiferentemente de su género y estado civil.

Respecto a las técnicas e instrumentos de recolección de datos, se emplea la encuesta mediante un cuestionario estructurado por doce (12) ítems con respuestas de selección múltiples con escala de Likert, utilizando cuatro alternativas de respuesta: nunca (1), casi nunca (2), casi siempre (3), siempre (4), dándole un valor del 1 al 5 a las respuestas negativas y del 5 al 1 a las positivas, validado por el juicio de diez (10) expertos en el área de gerencia en las organizaciones, con una confiabilidad de $r t t=0,857$ calculada con el método Alfa Cronbach( $\alpha$ ), utilizando el programa IBM SPSS Statistics Base, versión 22.0. Posterior a la fase de recolección de datos, se avanzó con el análisis de datos, donde se codificaron y asignaron previamente un valor numérico, para tabular adecuadamente los datos arrojados de acuerdo con la estadística descriptiva, agrupando estos datos en frecuencias absolutas y relativas, representada con una media aritmética y desviación estándar, según la cantidad de casos que contenían una característica y/o rasgo determinado, dada por las opciones de respuestas antes señaladas.

\section{RESULTADOS Y DISCUSIÓN}

En este apartado se dan a conocer los resultados y su discusión, esto producto del análisis efectuado por el estudio descriptivo de la variable en cuestión, y que a su vez permite hacer un contraste y comparación de los resultados con los refrentes teóricos e investigaciones reportadas. En la tabla 2 se muestran que el $59.1 \%$ de los coordinadores de postgrado de la universidad del estado de Zulia encuestados manifestó que siempre están presentes los indicadores que componen la integración a una estructura organizacional, el $34 \%$ expuso que casi siempre, el $6.9 \%$ casi nunca y el $0 \%$ nunca, denotando una tendencia positiva, con una media aritmética de la muestra de 3.5 y desviación estándar de 0.4 , el indicador con mayor puntaje fue el ideológico, seguidamente de lo orgánico-estructural, posteriormente el normativo, tecnológico, siendo el menos favorecido el psico-social.

Tabla 2: Integración de la estructura organizacional de las coordinaciones de postgrados en las universidades del estado Zulia

\begin{tabular}{|l|c|c|c|c|c|c|}
\hline \multirow{2}{*}{ Indicador } & Nunca & Casi nunca & Casi siempre & Siempre & $\dot{x}$ & $\Sigma$ \\
\cline { 2 - 7 } & Coordinador & Coordinador & Coordinador & Coordinador & Coordinador & Coordinador \\
\hline Ideológico. & 0.0 & 0.0 & 25 & 75 & 3.7 & 0.4 \\
\hline Orgánico-Estructural. & 0.0 & 0.0 & 27.2 & 72.8 & 3.7 & 0.3 \\
\hline Tecnológico. & 0.0 & 0.0 & 39.7 & 60.3 & 3.6 & 0.4 \\
\hline Psico-social. & 0.0 & 34.3 & 41.7 & 24 & 2.9 & 0.7 \\
\hline Normativo. & 0.0 & 0.0 & 36.2 & 63.8 & 3.6 & 0.3 \\
\hline Promedio. & 0.0 & 34.3 & 169.8 & 295.9 & 17.5 & 2.1 \\
\hline Porcentaje. & $0.0 \%$ & $6.9 \%$ & $34 \%$ & $59.1 \%$ & 3.5 & 0.4 \\
\hline
\end{tabular}


Al detalle, se puede decir que el indicador ideológico, el $75 \%$ encuestados manifestó que siempre están presentes los atributos que permiten su integración a la estructura organizacional, el $25 \%$ manifestó casi siempre, el $0 \%$ casi nunca y el $0 \%$ nunca. Continuando con el indicador orgánico-estructural, se tiene que el $72.8 \%$ de los coordinadores manifestaron que siempre están presentes en la estructura organizacional, el $27.2 \%$ manifestó que casi siempre, el $0 \%$ casi nunca y el $0 \%$ nunca. De la misma manera, el indicador tecnológico arrojó que el $60.3 \%$ de los coordinadores consultados en la muestra manifestaron que siempre están presentes los atributos que permiten su integración a la estructura organizacional, el 39.7\% manifestó casi siempre, el $0 \%$ casi nunca y el $0 \%$ nunca. Al revisar el indicador psico-social, el $41.7 \%$ de los coordinadores manifestaron que casi siempre están presentes los atributos que permiten su integración a la estructura organizacional, el 34.3\% manifestó casi nunca, el $24 \%$ siempre y el $0 \%$ nunca, y el indicador normativo arrojó que el $63.8 \%$ de los coordinadores los postgrados de las Universidades del estado Zulia consultados en la muestra manifestaron que siempre están presentes los atributos que permiten su integración a la estructura organizacional, el $36.2 \%$ manifestó casi siempre, el $0 \%$ casi nunca y el $0 \%$ nunca.

Después de conocer los resultados obtenidos, se puede evidenciar que la integración a la estructura organizacional de las coordinaciones de postgrados se está llevando a cabo mediante los indicadores antes expuestos, para el indicador ideológico, caracterizado por los recursos simbólicos que orientan y/o determinan las conductas tanto particulares como grupales, está incluido en las instituciones estudiadas. Para el indicador orgánico-estructural, comprendido por la infraestructura organizacional y a la forma como ésta fue acomodada, está incluido en las instituciones estudiadas. En cuanto a lo tecnológico, comprende los recursos de los procesos instrumentales de la organización. El indicador psicosocial, hace alusión a las interrelaciones interpersonales en la institución y sus grupos; en cuanto al normativo, forma parte de los preceptos y reglamentaciones que pretenden normar la actividad misma de la organización. Dichos resultados contrastan con lo propuesto por Belloso (2013), Bello-Pintado y Bianchi (2018) y Ramírez et al., (2020), quienes plantean que una organización es un sistema sociotécnico abierto, incluyendo los fines y valores, técnico, psicosocial, estructural y administrativo. Los fines y valores se refiere al objetivo que debería conseguir una organización. Por su lado, investigaciones efectuadas por Holbeche (2018), Sukier et al., (2020), y Sergeeva et al., (2021), coinciden con los resultados antes expuestos, en cuanto a lo psicosocial, donde está incluido por un conjunto en relación, encierra la conducta personal, motivación, interacción de funcionalidad y postura, dinámicas de conjuntos y participación. Finalmente, el indicador administrativo engloba a la organización al relacionarla con su ambiente, fijar las metas, desarrollar planes estratégicos y operativos, diseñar la composición y entablar procesos de control.

Al valorar los hallazgos reportados en la integración de la estructura organizacional de las coordinaciones de postgrados en las universidades del estado Zulia Ideológico, según lo orgánico-estructural, tecnológico, psicosocial, y normativo, son similares a las investigaciones como las de Ryttberg y Geschwind (2021), Annía et al., (2019), e Inciarte (2012), donde se colocan de manifiesto la articulación de la variable de estudio para integrar coherentemente las organizaciones, y que estas respondas a las demandas del entorno, propiciando en su quehacer laboral una gestión administrativa estratégica adaptadas a los cambios del medio, generando sustentabilidad cónsonas a su realidad, con perspectivas de nuevos mercados y amplitud de sus servicios, donde el aprendizaje significativo sea la prioridad en sus principios corporativos.

\section{CONCLUSIONES}

Posterior a la presentación de los resultados y a su discusión, se concluye que: (1) la estructura organizacional en las coordinaciones de postgrados de las universidades del estado Zulia, integran la cultura de la institución y las dimensiones de su doctrina, estandarizando el comportamiento organizacional como respuesta operante en la consolidación de metas, impactando de forma orgánico-estructural la administración estratégica, con carácter normativo que para la orientación de sus personas y se apoye en los recursos tecnológicos, a fin de generar un impacto psico-social adecuado, para la generación de valor sustentable; (2) aunque la integración de la estructura organizacional agrupa los indicadores ideológico, orgánico-estructural, tecnológico, psico-social y normativo, estos se pueden tratar de manera individual como estrategia y sello distintiva de las universidades, a fin de generar una propuesta de valor que garantice la identificación del cliente ante el mercado y a su vez se convierta en un referente específico, y se pueda ver posteriormente como una cadena de valor en el sistema integral de gestión institucional; (3) el hecho que se presente un desbalance de los indicadores de la estructura organizacional, genera un impacto psico-social en las personas de la comunidad académica, redundando en las desmejoras de los principios interinstitucionales y afectando negativamente en las estrategias del negocio; (4) la teoría en referencia confirman el origen de nuevos indicadores emergentes de la variable de estudio en cuestión, dando respuesta a los distintos cambios inestables del mercado, apuntando a la economía de escala, virtualización, talento humano verde, valor de lo intangible, generación de valor en las organizaciones y potencialización de las competencias genéricas, para adaptarse a los retos estructurales de las universidades, envueltas en la competitividad; (5) las perspectivas de la estructura organizacional, indiferentemente de la naturaleza de las instituciones, están experimentando nuevas maneras de hacer las cosas, con miras a lo glocal y sustentable, como mecanismo de respuesta para la gestión, apropiación y transferencia de conocimiento, con un enfoque 
integral, democrático, tecnológico y flexible, vistas en un ecosistema de cocreación e innovación, centradas en el desarrollo del individuo.

\section{REFERENCIAS}

Annía, M.E., Villalobos, J., Ramírez, R.I., y Ramos, Y., Capacidades dinámicas frente a la incertidumbre: una mirada desde la gestión universitaria. Revista Venezolana de Gerencia (RVG), 24(88), 1357-1372 (2019)

Arias, F. G., El proyecto de investigación, $6^{a}$ Ed., 1-143, Editorial episteme, C.A., Caracas, República Bolivariana de Venezuela (2012)

Bello-Pintado, A., y Bianchi, C., Educational diversity, organizational structure and innovation performance: evidence from uruguayan industry. https://doi.org/10.4067/S0718-52862018000200203, Estudios de Economía, 45(2), 203-229 (2018)

Belloso, R., A study of the types of organizational structure in venezuelan university institutes. Revista Electrónica de Investigación Educativa, 15(3), 53-67 (2013)

Braßler, M., The role of interdisciplinarity in bringing PBL to traditional universities: opportunities and challenges on the organizational, team and individual level. https://doi.org/10.14434/jijpbl.v14i2.28799, Interdisciplinary Journal of Problembased Learning, 14(2), 1-14 (2020)

Brunet, F., Malas, K., y Fleury, D., A model of an agile organization designed to better manage the COVID-19 crisis. https://doi.org/10.1177/0840470420980478, Healthcare Management Forum, 34(2), 115-118 (2021)

Dixon-Fowler, H., O'Leary-Kelly, A., Johnson, J., y Waite, M., Sustainability and ideology-infused psychological contracts: an organizational- and employee-level perspective. https://doi.org/10.1016/j.hrmr.2019.100690, Human Resource Management, 30(3), 100690 (2020)

Gomera, A., Antúnez, M., y Villamandos, F., Universities that learn to tackle the challenges of sustainability: case study of the university of cordoba (Spain). https://doi.org/10.3390/su12166614, Sustainability, 12(16),1-20 (2020)

Hernández, R., Fernández, C., y Baptista, M., Metodología de la investigación, 6ª Ed., 001-607, McGraw - Hill, Distrito Federal, México (2014)

Holbeche, L. S., Organisational effectiveness and agility. https://doi.org/10.1108/JOEPP-07-2018-0044, Journal of Organizational Effectiveness, 5(4), 302-313 (2018)

Inciarte, A., Gerenciar el cambio en la formación de postgrado en Venezuela. Revista Venezolana de Gerencia (RVG), 7(17), 49-63 (2012)

Ipek, Ö. F., y Karaman, A., Systemic change in a higher education institution: inquiring into organizational and instructional transformation. https://doi.org/10.1007/s11213-019-09516-6, Systemic Practice and Action Research, 34, 109-125 (2021)

Marín, R., Francis, H., y otros 3 autores., Self-evaluation system and use of information technology in higher education in the Republic of Ecuador. RISTI - Revista Ibérica de Sistemas e tecnologías de Informacao, 31, 109-120 (2020)

Menon, S., y Suresh, M., Factors influencing organizational agility in higher education. https://doi.org/10.1108/BIJ-042020-0151, Benchmarking, 28(1), 307-332 (2021)

Mihaljević, S., Exploring change in higher education: a case of doctoral education reforms.

https://doi.org/10.1504/IJMIE.2020.103828, International Journal of Management in Education, 14(1), 67-93 (2020)

Modenov, A., y Vlasov, M., Organizational structure and economic security of an enterprise. Espacios, 39(39), 1-22 (2018)

Ramírez, R. I., Espindola, C. A., Ruiz, G. I., y Hugueth, A. M., Gestión del talento humano: análisis desde el enfoque estratégico. http://dx.doi.org/10.4067/S0718-07642019000600167, Información Tecnológica, 30(6), 167-176 (2019)

Ramírez, R. I., Lay, N. D., y Sukier, H. B., Gerencia estratégica para la gestión de personas del sector minero de Venezuela, Colombia y Chile. http://dx.doi.org/10.4067/S0718-07642020000100133, Información Tecnológica, 31(1), 133-140 (2020)

Ríos, J. D., Crissien, T. J., y otros cuatro autores., Current state of agricultural units: as a phenomenon and complexity. Utopía y Praxis Latinoamericana. Revista internacional de filosofía iberoamericana y teoría social, 25(2), 452-468 (2020)

Ríos, J. D., Ramírez, R. I., y otros tres autores., Elements, resources and capacities of agricultural production units: from a thoughtful analytical approach. En Utopía y Praxis Latinoamericana. Revista internacional de filosofía iberoamericana y teoría social, 24(6), 407-419 (2019)

Ryttberg, M., y Geschwind, L., Organising professional support staff at higher education institutions: a multidimensional, continuous balancing act. https://doi.org/10.1007/s11233-020-09064-y, Tertiary education and management, 27, 47-58 (2021)

Saarikko, T., Westergren, U. H., y Blomquist, T., Digital transformation: five recommendations for the digitally conscious firm. https://doi.org/10.1016/j.bushor.2020.07.005, Business Horizons, 63(1), 825-839 (2020) 
Salmela, M., MacLeod, M., y Munck, J., Internally incentivized interdisciplinarity: organizational restructuring of research and emerging tensions. https://doi.org/10.1007/s11024-020-09431-4, Minerva, 1(1),1-23 (2021)

Santangelo, J., Hobbie, L., y otros 4 autores., The (STEM)2 network: a multi-institution, multidisciplinary approach to transforming undergraduate STEM education. https://doi.org/10.1186/s40594-020-00262-z, International Journal of STEM Education, 8(1), 1-3 (2021)

Sergeeva, M., Sokolova, A., y otros 3 autores., Technology of forming a multicomponent organizational structure of a continuous pedagogical education system. https://doi.org/10.20511/pyr2021.v9nSPE2.991, Propósitos y representaciones, 9(2), 2-11 (2021)

Sukier, H. B., Ramírez, R. I., y otros cuatro autores., Strategic Management of human talent from a sustainable approach. Opción. Revista de ciencias humanas y sociales, 36(91), 929-953 (2020a)

Sukier, H. B., Ramírez, R. J., Ramírez, R. I., y Lay, N. D., Administración estratégica en el sector salud desde el enfoque organizacional. Revista Venezolana de Gerencia (RVG), 25(04), 206-221 (2020)

Tamayo, M., Técnicas de la investigación, 3ª Ed., 001-435, Panapo S, A, Madrid, España (2012)

Villalobos, J V., Ramírez, R. I., y Diaz, L., Bioética y biopoder: perspectivas para una praxis pedagógica desde la ética de Álvaro Márquez-Fernández. Utopía y Praxis Latinoamericana. Revista internacional de filosofía y teoría social, 24(87), 65-77 (2019)

Villalobos, J. V., y Ramírez, R. I., El derecho a la autobiografía: dimensión ius-filosófica desde la perspectiva de H. Arendt y P. Ricoeur. Opción. Revista de ciencias humanas y sociales, 34(18), 1012-1587 (2018) 
